Leading Article

\title{
The Indispensable Ally - The Pathologist's Pre-eminent Role in Renal Medicine through the ages.
}

\author{
Chula Herath MBBS, MD, FRCP(Lond), FCCP \\ Consultant Nephrologist, General Hospital Sri Jayewardenepura, Sri Lanka.
}

In the mid-17th century, Italian physician Marcello Malpighi first described the microscopic anatomy of human kidneys using an early microscope developed by the English polymath Robert Hooke. However, it was not until the mid-18th century that microscopic pathology was used as a useful tool for diagnosing renal disease. William Bowman, an English physician (1816-1892) improved on Malpighi's work and described the proximal nephron, a part of which carries his name- the Bowman's capsule [1]. Richard Bright (17891858) of London Guy's Hospital, often called the Father of Nephrology, elucidated the laboratory and clinical features of acute glomerulonephritis depending on macroscopic rather than microscopic descriptions [2]. Acute nephritic syndrome was called Bright's disease until the early nineteen seventies. The development of microscopic pathology accelerated in 19th century when first, the Swiss physiologist Gabriel Valentin devised a technique to cut thin slices to make slides in 1837[3]. Subsequently, his compatriot Edwin Klebs learned to embed tissue samples in paraffin [4]. The German physician Friedrich Henle (1809-1885), an eminent figure in the growth of modern medicine described the long tubular segment which follows the proximal tubule which was henceforth named the loop of Henle [5]. Parallel to the development of pathology was the gradual unravelling of the "milieu interior" by Carl Ludwig (1816-1895), Claude Bernard (1813-1878) and later Homer Smith (1895-1962) [6].

Until the early part of the twentieth century, there was little attempt to correlate microscopic renal pathology with laboratory and clinical features of renal disease. Theodor Fahr (18771945) of Hamburg was possibly the first modern renal pathologist. His accurate histological drawings were often mistaken for photographs [7]. He together with Franz Volhard, a physician from Mannheim combined their skills to produce a text-book of renal pathology where for the first-time clinical features were correlated with histology [8]. This was probably the first collaboration between a pathologist and a physician in nephrology. One of their drawings entitled "lipoid nephrosis with glomerular degeneration" illustrates what we now recognize as focal segmental glomerulosclerosis (FSGS) with attention to podocyte swelling and depletion accompanied by hyalinosis and capsular adhesion, even before these cells or lesions were understood [7]. Sir Arthur Ellis (1883-1966) initially a clinical chemist, who later trained in pathology and then in internal medicine, had the ideal foundation to correlate clinical chemistry, pathology and clinical manifestations of renal disease. His classic paper published in Lancet in 1942 described clinicopathological correlations of 600 patients with Bright's disease [9].

Even well into the mid-20th century, all renal pathology was done on post-mortem or postsurgical specimens. The first live renal biopsy was done in 1951 by Poul Iverson and Claus Brun in Copenhagen [10]. The technique was improved and popularized in the United States and thus began the fruitful collaboration between clinicians and pathologists to elucidate the evolution of renal disease and its clinicopathology correlations. Immunofluorescence microscopy (IFM) was first utilized on renal 
biopsy tissue by Mellors in 1955[11], and the first descriptions of electron microscopy of renal ultrastructure was published in 1957 [12]. Conrad Pirani and his renowned Chicago team were pioneers in using electron microscopy for renal pathology diagnosis. A land-mark textbook of renal pathology was published in 1966 by Robert Heptinstall of Johns Hopkins University which is still known as "Heptinstall's Pathology of the Kidney".

It was not until 1960 that the term "nephrology" was coined by the first chairman of the International Society of Nephrology (ISN) Jean Hamburger [7]. In 2020, a nephropathologist was elected as the President of ISN for the second time when Agnus Fogo was elected to this prestigious post which indicates the esteem nephropathology is held by the community of nephrologists.

Collaborations between nephrologists and pathologists has illuminated many previously poorly-understood areas in nephrology. Even into the early 21st century, the etiology of the three main idiopathic nephrotic syndromes minimal change disease, primary FSGS and membranous nephropathy- were still unclear. Primary membranous nephropathy is now understood to be largely mediated by antibody to phospholipase A2 receptor 13 and some antigens such as cationic bovine serum albumin 43 and neutral endopeptidase. Membranoproliferative glomerulonephritis (MPGN) was previously classified as types I, II, and III based on the location and microscopic appearance of deposits, rather than their immune composition. It is now understood that subsets of type I and type III MPGN that contain C3 only, without immunoglobulin, are mediated by inherited or acquired dysregulation of the alternative complement pathway. MPGN now falls under the umbrella of C3 glomerulopathies [14]. Staining for IgG subtypes has recognized IgG4associated autoimmune interstitial nephritis associated with multi-organ involvement [15]. Identification of complement component C4d deposition in peritubular capillaries has revolutionized the diagnosis of kidney transplant antibody-mediated rejection.
Both nephrology and renal pathology have been relatively slow to develop in Sri Lanka. This may be because nephrology was recognized as a subspecialty relatively late in 1999 . The first searchable renal pathology case report seems to have appeared in 1970 [16]. In the 1990s, when the interest in nephropathology was still scant, pioneering work of Preethika Angunawela of University of Colombo led to several publications on IgA nephropathy, Lupus nephritis and on the use of immunohistochemistry in renal pathology, as well as a short textbook of renal pathology for medical students $[17,18]$. Emergence of a new form of chronic kidney disease known as chronic kidney disease of unknown cause (CKDu) has initiated a renaissance in renal pathology. Many publications on the pathology of this devastating disease have appeared since the first reports in 2011 [19]. Contributions of Neelakanthi Ratnatunga of University of Peradeniya in elucidating the renal ultrastructure in CKDu have been significant [20]. Techniques in native kidney and transplant biopsy preparations and stains for light microscopy have made significant strides but immunofluorescence and immunohistochemistry are still limited to a few teaching hospitals, while it's not possible to get routine electron microscopy in renal biopsy specimens.

So, what needs to be done to optimize this vital partnership between these two specialties in Sri Lanka? Firstly, both pathologists and nephrologists need to have a sound knowledge of each other's specialty. Weekly in-hospital team meetings and collaborative meetings with other centers are an essential prerequisite for this. Each renal biopsy should be discussed between the two teams before a final report is issued. Acquisition of facilities for making virtual slides would greatly improve telemedicine services to peripheries. Secondly, pathology and nephrology societies should pool resources for creating a renal biopsy registry, first at provincial level and then at national level. For this, an international coding practice adapted for Sri Lanka should be adhered to for diagnosis and nomenclature, so there will be consistency in 
reporting across different units. Thirdly, much more basic research and publications should be encouraged from the trainee levels. Aforementioned renal biopsy registry will be an invaluable asset for researchers. Finally, nephrologists should assist pathologists to obtain funding for the essential facilities such as special stains, IFM and electron microscopy. They should use the unfair advantage clinicians generally have in acquiring funds from policymakers and administrators to improve their pathology departments, albeit for selfish reasons.

Danish author Karen Blixen of "Out of Africa" fame once wrote "What is man, when you come to think upon him, but a minutely set, ingenious machine designed to turn, with infinite artfulness, the red wine of Shiraz into urine?". The kidney, being the true ingenious machine, which turns red wine to urine has yielded some of its secrets to the toil of devoted scientists. The pathologist has much to do in unravelling the remaining secrets as it's often felt that the keys to "uncertain causes" likely lie in pathology. To the nephrologist this is truly the indispensable ally.

\section{References}

1. Bowman W (1842) On the structure and use of the Malpighian bodies of the kidney, with observations on the circulation through that gland. Philos Trans R Soc 132:57-80

2. Bright $R$ (1827) Reports of medical cases selected with a view of illustrating the symptoms and cure of disease by a reference to morbid anatomy. Longman, Rees, Orme and Green, London

3. Valentin G (1837) Repertorium fuer anatomie und physiologie. Huber, Bern

4. Klebs TAE (1870) Handbuch der pathologischen anatomie. August Hirschwald, Berlin, pp 644-648

5. Henle J (1862) Zur Anatomie der Niere. Dieterichschen Buch-handl, Goettingen

6. Weening JJ, Jennette JC. Historical milestones in renal pathology. Virchows
Arch. 2012 Jul;461(1):3-11. doi: 10.1007/s00428-012-1254-7. Epub 2012 Jun 3

7. D'Agati VD, Mengel $M$. The rise of renal pathology in nephrology: structure illuminates function. Am J Kidney Dis. 2013 Jun;61(6):1016-25. doi: 10.1053/j.ajkd.2012.12.019. Epub 2013 Feb 12.

8. Volhard F, Fahr T (1914) Die Brightsche Nierenkrankheit. Klinik, Pathologie und Atlas. Julius Springer, Berlin

9. Ellis A (1942) Natural history of Bright's disease. Clinical, histo-logical and experimental observations. Lancet 1:1-7

10. Iversen P, Brun C (1951) Aspiration biopsy of the kidney. Am J Med 11:324330.

11. Mellors RC (1955) Histochemical demonstration of the in vivo localization of antibodies: antigenic components and the pathogen-esis of glomerulonephritis. J Histochem Cytochem 3:284-289.

12. Farquhar MG, Vernier RL, Good RA (1957) An electron micro-scope study of the glomerulus in nephrosis, glomerulonephritis and lupus erythematosus. J Exp Med 106:649-660.

13. Beck LH Jr, Bonegio RG, Lambeau G, Beck DM, Powell DW, et al. J. M-type phospholipase A2 receptor as target antigen in idiopathic membranous nephropathy. N Engl J Med. 2009 Jul 2;361(1):11-21. doi: 10.1056/NEJMoa0810457.

14. Sethi S, Fervenza FC. Membranoproliferative glomerulonephritis--a new look at an old entity. N Engl J Med. 2012 Mar 22;366(12):1119-31. doi: 10.1056/NEJMra1108178.

15. Raissian $Y$, Nasr SH, Larsen CP, Colvin RB, Smyrk TC, Takahashi N, et al. Diagnosis of IgG4-related tubulointerstitial nephritis. J Am Soc Nephrol. 2011 Jul;22(7):1343-52. doi: 10.1681/ASN.2011010062. 
16. Varagunam T, Panabokke RG. Bilateral cortical necrosis of the kidneys following snakebite. Postgrad Med J. 1970 Jul;46(537):449-51. doi: 10.1136/pgmj.46.537.449.

17. Angunawela $P$, Sheriff R. Diagnosis of IgA nephropathy by immunohistochemical methods. Journal of the Ceylon College of Physicians. 1992; 25:52-55.

18. Angunawela P. Diagnosis of glomerulonephritis using light microscopical, immunological and ultrastructural techniques. Journal of the Ceylon College of Physicians. 1995;28 (1), 46-49.
19. Athuraliya NT, Abeysekera TD, Amerasinghe $\mathrm{PH}$, Kumarasiri $\mathrm{R}$, Bandara $\mathrm{P}$, Karunaratne $\mathrm{U}$, Milton $\mathrm{AH}$, Jones $\mathrm{AL}$. Uncertain etiologies of proteinuricchronic kidney disease in rural Sri Lanka. Kidney Int. 2011 Dec;80(11):1212-21. doi: 10.1038/ki.2011.258. Epub 2011 Aug 10.

20. Nanayakkara S, Komiya T, Ratnatunga N, Senevirathna ST, et al. Tubulointerstitial damage as the major pathological lesion in endemic chronic kidney disease among farmers in North Central Province of Sri Lanka. Environ Health Prev Med. 2012 May;17(3):213-21. doi: 10.1007/s12199-011-0243-9. 